\title{
Periorbitales Kontaktekzem durch das Cholinergicum Aceclidin
}

\section{Kati Mayer \\ Ruthild Linse \\ W. Harth}

\section{Periorbital Eczema through the Cholinergicum Aceclidin}

\section{Zusammenfassung}

Neben atopischen Augenekzemen sind Kontaktallergien die häufigsten Ursachen rezidivierender periorbitaler Ekzeme. Über Kontaktekzeme im Zusammenhang mit der Glaukomtherapie, insbesondere durch die Anwendung von Cholinergika, liegen nur wenige Veröffentlichungen vor. Es wird eine 69-jährige Patientin mit einem periorbitalen Ekzem vorgestellt, die aufgrund eines Glaukoms über mehrere Jahre mit diversen Antiglaukomatosa behandelt wurde. In der allergologischen Abklärung findet sich im Epikutantest eine positive Testreaktion auf das Cholinergikum Aceclidin (Glaukotat ${ }^{\circledR}$ ). Trotz der sehr selten auftretenden allergischen Reaktionen auf Cholinergika sollte besonders bei der exponierten Gruppe der Glaukompatienten die Rolle der Substanz als Allergen nicht unterschätzt werden.

\section{Abstract}

In addition to atopic eczema contact allergies are the main cause of recurring periorbital eczema. There are only few case reports about periorbital contact dermatitis due to topical therapy of glaucoma, especially of parasympathicomimetics causing contact eczema. We report about a 69-year old female patient with bilateral periorbital eczema. Because of a glaucoma she received several local antiglaucomatosa within the last years. The patchtest revealed an allergic reaction for Aceclidin (Glaucotat ${ }^{\circledR}$ ). Contact allergies due to topical cholinergica are only rarely found. However, their sentitization potential shouldn't be underestimated, especially in the group of glaucoma-patients.

\section{Einleitung}

Neben atopischen Augenekzemen sind Kontaktallergien die häufigste Ursache für rezidivierende periorbitale Ekzeme. Die häufigsten Allergene stellen die in Kosmetika enthaltenen Duftstoffe, Formaldehydharz, Amerchol und Chloramphenicol dar [1,6].

Über periorbitale Kontaktekzeme im Zusammenhang mit der Glaukombehandlung liegen bisher nur wenige Veröffentlichungen vor. Die Mehrzahl der durch Antiglaukomatosa hervorgerufenen Allergien ist auf die verwendeten Lösungsmittel, wie Benzalkoniumchlorid und Thiomersal, zurückzuführen. Auch über Kontaktallergien durch die Sympathikomimetika Phenylephrin, Epinephrin, Brimonidin und Apraclonidine wurde in Einzelfällen berichtet $[7,8,13]$. Es finden sich jedoch bislang keine Literaturhinweise über periorbitale Kontaktekzeme, die durch Parasympathikomimetika im Rahmen einer Glaukombehandlung aufge- treten sind. Anhand einer Kasuistik soll auf die Möglichkeit der Entstehung einer Kontaktallergie durch das Parasympathikomimetikum Aceclidin (Glaukotat ${ }^{\circledR}$ ) aufmerksam gemacht werden.

\section{Kasuistik}

\section{Anamnese}

Eine 69-jährige Patientin leidet seit Monaten unter einer beidseitigen periorbitalen Rötung, leichter Schwellung und massivem Juckreiz. Wegen eines seit Jahren bestehenden Glaukoms war die Patientin auf Glaukotat ${ }^{\circledR}$ Augentropfen (Aceclidinhydrochlorid) eingestellt worden. Vorher waren Xalatan ${ }^{\circledR}$ Augentropfen (Latanoprost) und Trusopt ${ }^{\circledR}$ Augentropfen (Dorzolamid) verwendet worden (Abb.1). 


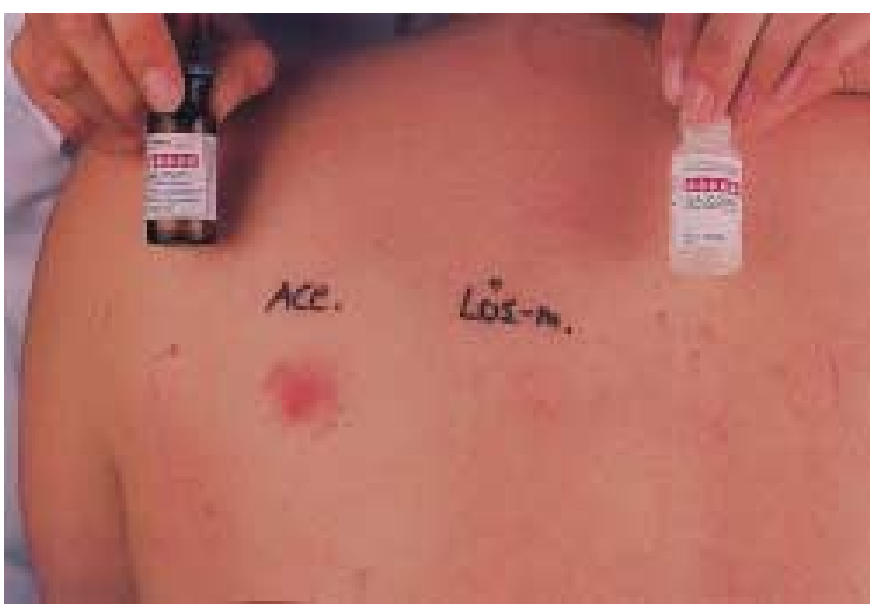

Abb. 2 Im Epikutantest zweifach positive Testreaktion auf Aceclidin nach 24 und 48 h, keine Reaktion auf das verwendete Lösungsmittel.

\section{Aufnahmebefund}

Periorbital beidseits finden sich eine relativ scharf begrenzte Rötung, eine diskrete Schwellung und eine Vergröberung des Hautreliefs. Es besteht eine atopische Disposition.

\section{Untersuchungsbefunde}

Unter dem Verdacht auf ein allergisches Kontaktekzem auf die bislang eingetropften Antiglaukomatosa führten wir neben der Testung der Standardreihe des Epikutantestbogens sowie der augenspezifischen Wirkstoffe und Konservierungsmittel von Hermal eine Epikutantestung der verwendeten Augentropfen nach vorherigem zweimaligen Stripping der Haut mit Tesafilm durch (Tab. 1 u. 2).

Die Ablesungen nach 48 und $72 \mathrm{~h}$ ergaben eine dreifach positive Reaktion auf Glaukotat ${ }^{\circledR}$ Augentropfen. Die Glaukotat ${ }^{\circledR}$ Augentropfen bestehen aus der Trockensubstanz, die Aceclidinhydrochlorid, Borsäure, Natriumtetraborat enthält. Das Lösungsmittel enthält Benzalkoniumchlorid, Borsäure, Natriumtetraborat, gereinigtes Wasser. Im Epikutantest ergaben sich nach 48 und $72 \mathrm{~h}$ zweifach positive Reaktionen auf die Trockensubstanz, die Aceclidinhydrochlorid enthält. Das getestete Lösungsmittel zeigte keine positive Reaktion (Abb. 2).

\section{Verlauf}

Die Patientin wurde im weiteren Verlauf auf ein anderes Antiglaukomatosum eingestellt. Hierunter sowie der externen Behandlung mit OTC-Augensalbe kam es zur raschen und vollständigen Abheilung der Hautveränderungen.

\section{Diskussion}

Patienten mit Lidekzemen stellen den Dermatologen oftmals vor diagnostische und therapeutische Schwierigkeiten, da zum einen zumeist ein chronischer Verlauf mit hohem subjektiven Leidensdruck des Patienten besteht, zum anderen mögliche Allergene insbesondere in Kosmetika und Ophthalmologika - seitens des Patienten nicht angegeben werden, sei es durch Vergessen oder da die Patienten unter den verwendeten Substanzen nicht den Auslöser vermuten. Hier sind eine genaue Anamnese sowie die Kenntnis möglicher, auch seltener Allergene, unerlässlich.
Tab. 1 Testung der von der Patientin verwendeten Augentropfen

\begin{tabular}{lll}
\hline & $\mathbf{4 8 h}$ & $\mathbf{7 2 h}$ \\
\hline Xalatan $^{\circledR}$ & $\varnothing$ & $\emptyset$ \\
Trusopt $^{\circledR}$ & $\emptyset$ & $\emptyset$ \\
Claukotat $^{\circledR}$ & +++ & +++ \\
\hline
\end{tabular}

Tab. 2 Testung der Einzelsubstanzen der Glaukotat ${ }^{\circledR}$ Augentropfen

\begin{tabular}{lcc}
\hline & $\mathbf{4 8 ~}$ & $\mathbf{h} \boldsymbol{h}$ \\
\hline $\begin{array}{l}\text { Glaukotat }{ }^{\circledR} \text { Trockensubstanz (Aceclidin- } \\
\text { hydrochlorid, Borsäure, Natriumtetraborat) }\end{array}$ & ++ & ++ \\
$\begin{array}{l}\text { Glaukotat }{ }^{\circledR} \text { Lösung (Benzalkoniumchlorid, } \\
\text { Borsäure, Natriumtetraborat) }\end{array}$ & $\varnothing$ & $\varnothing$
\end{tabular}

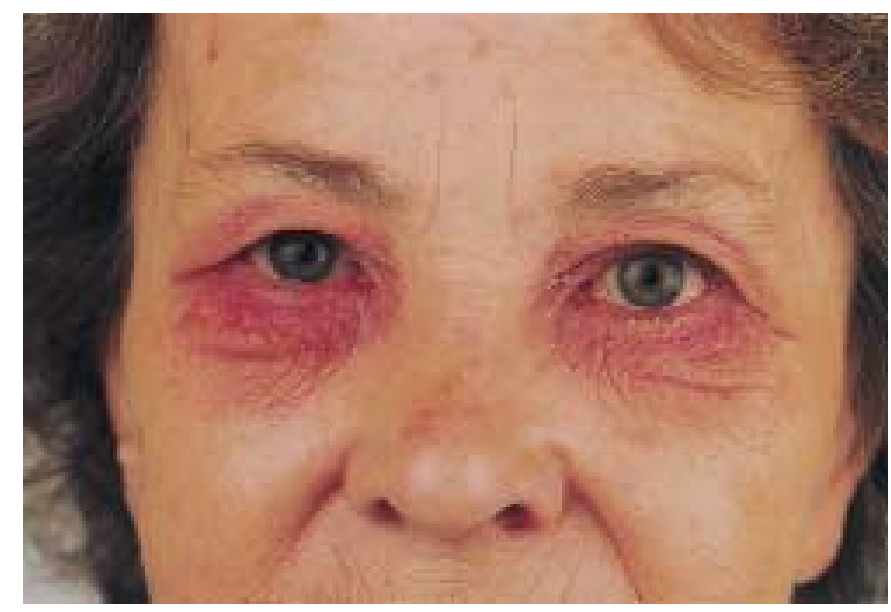

Abb. 1 Seit mehreren Monaten bestehendes periorbitales Ekzem mit Rötung, Schuppung und Lichenifikation.

Die häufigste Ursache für rezidivierende Lidschwellungen stellen durch Kosmetika und ophthalmologische Lokaltherapeutika verursachte Kontaktekzeme dar. Innerhalb der Gruppe der Ophthalmologika führen neben den relativ häufig sensibilisierenden Antibiotika wie Chloramphenicol und Gentamycin insbesondere die verwendeten Konservierungsmittel, z. B. Benzalkoniumchlorid und Thiomersal zu periorbitalen Kontaktekzemen. Im Zusammenhang mit der Glaukombehandlung werden vor allem Sensibilisierungen gegenüber diversen Betablockern (Levobunolol, Timolol, Befunolol, Careolol) aufgeführt $[12,14]$.

Blum [8] berichtet über eine durch Phenylephrin, einem Sympathikomimetikum, hervorgerufene allergische Reaktion, die in der Allergologie bekannt ist, aber relativ selten beobachtet wird. In diesem Zusammenhang stellt Blum die Vermutung auf, dass die Häufigkeit einer Sensibilisierung auf Sympathikomimetika höher ist, als bisher in der Literatur berichtet. Diese Vermutung wird durch 2 aktuelle Fallbeschreibungen über allergische Kontaktekzeme auf die Sympathikomimetika Apraclonidin und Brimonidin bestätigt $[10,11]$. Über allergische Kontaktekzeme 
durch Parasympathikomimetika (Cholinergika) finden sich in der Literatur bislang keine Hinweise. In diesem Kontext berichten wir über ein durch ein Cholinergikum (Aceclidin) hervorgerufenes periorbitales Kontaktekzem.

Cholinergika finden breite Anwendung in der Glaukomtherapie zur Senkung des Augeninnendruckes. Aceclidin (Glaukotat ${ }^{\circledR}$ ) ist ein synthetisches Parasympathikomimetikum, das zur lokalen Behandlung eines Glaukoms zur intraokulären Drucksenkung angewendet wird. Es handelt sich um ein an Position 3 acetyliertes Chinudinmolekül und stellt chemisch ein Razemat dar, dessen aktive Form das (+)-Isomer ist [2,3].

In Glaukotat ${ }^{\circledR}$ Augentropfen liegt das Aceclidin als Hydrochlorid vor. Die gebrauchsfertige Lösung enthält Aceclidin in lyophilisierter Form. Die Zubereitung erfolgt zur Verhinderung eines Aktivitätsverlustes des Antiglaukomatosums erst durch den Patienten.

Der Wirkstoff in Form einer Trockensubstanz und das Lösungsmittel werden dementsprechend getrennt aufbewahrt. Aceclidin wird seit vielen Jahren zur Behandlung aller Glaukomformen mit guter Verträglichkeit eingesetzt. In Anwendungsbeobachtungen in Form klinischer Studien wurden neben dem Ausmaß der gewünschten Drucksenkung auch die Nebenwirkungen des Medikamentes untersucht [2-5]. Allergische Reaktionen wurden von den Autoren bei der Untersuchung an 1211 Augen nur bei 0,32\% der Patienten beobachtet, diese jedoch nicht näher charakterisiert.

In Einzelfällen wird zu Beginn der Behandlung eine Hyperämie der Bindehaut beschrieben. Diese klingt in der Regel rasch wieder ab und ist von einer allergischen Reaktion, wie sie im vorliegenden Fall aufgetreten ist, abzugrenzen.

Im Rahmen der allergologischen Diagnostik wird vor der Epikutantestung von Ophthalmologika zur Darstellung einer Typ IVSensibilisierung die Durchführung eines Tesafilmabrisses empfohlen. Die von uns durchgeführte Epikutantestung wurde mit 2 Tesafilmabrissen vor dem Aufbringen der Testsubstanzen durchgeführt, was wir als ausreichend betrachten. Standardisierungen für diese Strippingmethode existieren bisher nicht.
Trotz der seltenen allergischen Reaktionen auf Cholinergika sollte die Bedeutung der Substanzgruppe als Allergen, besonders bei der exponierten Gruppe der Glaukompatienten, nicht unterschätzt werden. In diesem Sinne möchten wir auf diesen im Schrifttum erstmals berichteten Fall eines allergischen Kontaktekzems auf das Cholinergikum Aceclidin aufmerksam machen.

\section{Literatur}

${ }^{1}$ Manu S, Lewis FM, Gawlerodger DI. Facial dermatitis, an eye, lid dermatitis: a comparsium of patch test results and final diagnoses. Contact dermatitis 1996; 34: 140-141

${ }^{2}$ Rouher F. Klinische Studien mit Glaukotat bei verschiedenen Glaukomen. München: Chibret, 1970

${ }^{3}$ Pilz A et al. Experimentelle und klinische Untersuchungen mit Aceclidin (Glaukotat). Ophthalmologica 1974; 168: 376-391

${ }^{4}$ Riegel D, Leydhecker W. Erfahrungen mit Aceclidin bei der Behandlung von Glaucoma simplex. Klin Mbl Augenheilk 1967; 151: $882-885$

${ }^{5}$ Aceclidin zur Behandlung des Glaukoms - Erfahrungen aus der Praxis. Augenspiegel 11/91 (Sonderdruck)

${ }^{6}$ Ockenfels H, Seemann U, Goos M. Contact allergy in patients with periorbital eczema: an analysis of allergens. Dermatology 1997; 195: $119-124$

${ }^{7}$ Barber K. Allergic contact eczema to phenylephrine. Contact Dermatitis $1983 ; 7: 274-277$

${ }^{8}$ Blum A, Brunner C, Lischka G. Ödematöse Schwellung der Augenlider durch Kontaktallergie. Hautarzt 1998; 49: 651 -653

${ }^{9}$ Milpied B, Fleischmann M, Berre F, Sonrisse M, Litoux P. Another case of allergy contact dermatitis from phenylephrin in eyedrops. Contact Dermatitis 1988; 18: $146-147$

${ }^{10}$ Schuman JS, Horwitz B et al. A 1-year study of brimonidine twice daily in glaucoma and ocular hypertension. A controlled, randomized, multicenter clinical trial. Chronic Brimonidine Study Group. Arch Ophthalmol 1999; 117: 417-419

${ }^{11}$ Silvestre JF, Carnero L et al. Allergic contact dermatitis from apraconidine in eyedrops. Contact Dermatitis 2001; 45: 251

${ }^{12}$ Giordano-Labadie F, Leoittevin JP, Calix I, Bazex J. Contact allergy to beta blockaders in eye drops: cross allergy? Ann Dermatol Venereol 1997; 124: $322-324$

${ }^{13}$ Gaspari AA. Contact allergy to ophthalmic dipivalyl epinephrine hydrochloride: demonstration by patch testing. Contact Dermatitis 1993; 28: $35-37$

${ }^{14}$ Schultheiss E. Hypersensitivity to levobunolol. Derm Beruf Umwelt: 37: $185-186$ 